\title{
RBF-Based Representation of Volumetric Data: Application in Visualization and Segmentation
}

\author{
Yoshitaka Masutani \\ Image Computing and Analysis Laboratory, \\ Department of Radiology, University of Tokyo (UT-RAD/ICAL) \\ 7-3-1 Hongo Bunkyo-ku Tokyo 113-8655 Japan \\ tel. +81-3-5800-8666 ex.37418; fax. +81-3-5800-8935 \\ masutani-utradeumin.ac.jp
}

\begin{abstract}
A new scheme of data-driven segmentation is proposed, which is based on detection of object boundary, and volumetric pattern reconstruction as implicit function by using the detected object boundary and the radial basis functions (RBF). By using clinical X-ray CT data, applications in visualization of the pancreatic duct by MINIP of curved thin-slab and in liver segmentation are shown.
\end{abstract}

\section{Introduction}

Segmentation procedure is required in many fields of medical imaging. For example, in computer-assisted detection of lung nodule, segmentation of lung is an important process for limiting search area. In general, methods of segmentation are often categorized in two: data-driven, and model-based (mostly deformable models). The former is conventionally employed in many applications, based on thresholding, connected component analysis, math-morphology, etc. On the other hand, the latter is reported extensively, and is shown to be promising in several applications since SNAKES [Kass87] and other related methods based on deformable models were proposed. However, several limitations of deformable models are also found in cases shape variation of segmentation target is large, for example colon segmentation. For such cases, data-driven methods have still advantages and are employed [Masutani01]. However, in data-driven methods, it is hard to introduce a priori knowledge for eliminating misconnected objects. In this paper, a new segmentation method is proposed by extending the notion of radial basis functions (RBF), which were conventionally employed as surface shape interpolator / extrapolator. A new notion of non-boundary control points for representing a priori knowledge is also presented.

\section{Methods}

\subsection{Basic Theory of Radial Basis Functions for Construction of Volumetric Pattern}

Radial basis functions (RBF) are employed as a representation method of a solid and a surface interpolator / extrapolator since it was reported by several research groups [Savchenko95][Carr97][Turk99]. An interpolation / extrapolation process based on 
RBF is to construct an implicit function $s(\mathbf{x})$ by using several pairs of control point coordinates $\mathbf{x}_{i}$ and function value $s_{i}$ at $\mathbf{x}_{i}$, In this section, the theory of the RBF is briefly described.

\subsubsection{Construction of Volumetric Pattern as Implicit Function by Control Points}

An approximated implicit function $s(\mathbf{x})$ in $3 \mathrm{D}$ space $\left(\mathbf{x} \in \mathbf{R}^{3}\right)$ is expressed as

$$
s(\mathbf{x})=p(\mathbf{x})+\sum_{i=1}^{N} \lambda_{i} \phi\left(\left|\mathbf{x}-\mathbf{x}_{i}\right|\right)
$$

where $\mathbf{x}=(x, y, z)^{\mathrm{T}}$ is coordinates at arbitrary position in 3D space, $p(\mathbf{x})$ is a low degree polynomial function, $N$ is the number of control points, $\lambda_{i}$ is coefficient for control point, $\mathbf{x}_{i} \phi(r)$ is a basic function, and II is the Euclidean norm in $\mathbf{R}^{3}$. A basic function $\phi(r)$ is normally chosen from the well-known families of the spline functions of smoothing, such as the thin-plate, the Gaussian, the multi-quadratic, the bi-harmonic, the tri-harmonic, and so on. In this study, the bi-harmonic spline function, shown in equation (2) is employed for its energy minimization properties.

$$
\phi(r)=r
$$

In addition, the bi-harmonic spline function is one of the non-compactly supported functions, which are suitable for interpolation/extrapolation of irregular and nonuniform control points [Carr01]. For the bi-harmonic spline function as RBF, the polynomial function is

$$
p(\mathbf{x})=c_{1}+c_{2} x+c_{3} y+c_{4} z
$$

Therefore, the approximation process equals to computing the following values.

$$
\begin{aligned}
& \Lambda=\left(\lambda_{1}, \ldots, \lambda_{N}\right)^{\mathrm{T}} \\
& \mathbf{c}=\left(c_{1}, c_{2}, c_{3}, c_{4}\right)^{\mathrm{T}}
\end{aligned}
$$

However, it is clear that four degrees of freedom are still left in determining all these values only by using the equation (1) and $N$ pairs of control point coordinates $\mathbf{x}$ and function values $s$. To guarantee that $s(\mathbf{x})$ belongs to the Beppo-Levi space on $\mathbf{R}^{3}$, additional conditions of the orthogonality are required as follows.

$$
\sum_{i=1}^{N} \lambda_{i}=\sum_{i=1}^{N} \lambda_{i} x_{i}=\sum_{i=1}^{N} \lambda_{i} y_{i}=\sum_{i=1}^{N} \lambda_{i} z_{i}=0
$$

Finally, for the case bi-harmonic spline is chosen as RBF, the computing process is summarized as solving linear equations:

$$
\left(\begin{array}{cc}
\mathbf{A} & \mathbf{P} \\
\mathbf{P}^{\mathrm{T}} & \mathbf{0}
\end{array}\right)\left(\begin{array}{l}
\Lambda \\
\mathbf{c}
\end{array}\right)=\left(\begin{array}{l}
\mathbf{S} \\
\mathbf{0}
\end{array}\right)
$$

where $\quad A_{i, j}=\phi\left(\left|\mathbf{x}_{i}-\mathbf{x}_{j}\right|\right)=\left|\mathbf{x}_{i}-\mathbf{x}_{j}\right|, \quad \mathbf{S}=\left(s_{1}, \ldots, s_{N}\right)^{\mathrm{T}}$, 


$$
\mathbf{P}=\left(\begin{array}{cccc}
1 & x_{1} & y_{1} & z_{1} \\
& & \vdots & \\
1 & x_{N} & y_{N} & z_{N}
\end{array}\right)
$$

Then, a volume data set is simply reconstructed, by determining voxel value at $\mathbf{x}$ as $s(\mathbf{x})$.

\subsubsection{Configuration of Control Points with Boundary Points and Normals}

In constructing an implicit function representing an object shape that has complex boundary, normal vectors with boundary points are often necessary for stable construction of a function [Carr01]. As shown in Fig.1, in such cases, three control points with function values: $(\mathbf{x}, s)=(\mathbf{x}, 0),(\mathbf{x}+\mathbf{n}, 1),(\mathbf{x}-\mathbf{n},-1)$ are generated for a boundary point $\mathbf{x}$ with its normal $\mathbf{n}(\mathbf{x})$.
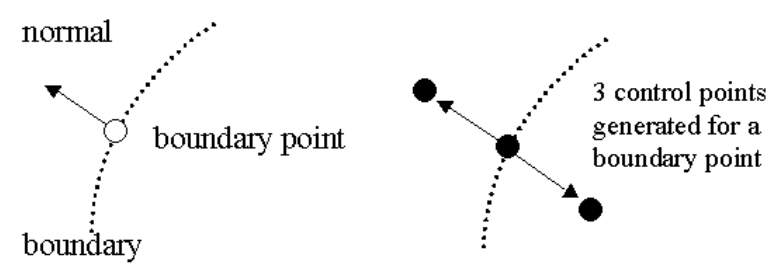

Fig. 1. Three Control Points Generated For A Boundary Point

\subsection{Application in Visualization by Forming Curved Thin Slab}

A simple application of RBF is determination of curved thin slab from sparsely distributed control points with normals. Fig.2 shows an example of the pancreatic duct visualization by minimum intensity projection. In this example, seven boundary points were interactively set on the pancreatic duct and all the normals of the boundary points are set to the normal of coronal plane. After computing the implicit function $s(\mathbf{x})$, the entire volume was cleared except for the voxels of which $s(\mathbf{x})$ is nearly zero, i.e. close to the boundary.

\subsection{Application in Segmentation of Objects in Volumetric Data}

By using RBF, segmentation of objects in volumetric data, is regarded as construction of an implicit function of which value is: 0 on the object boundary, positive inside the boundary, and negative outside the boundary. In other words, RBF-based segmentation, proposed in this paper, equals to defining boundary points of the object with their normals.

\subsubsection{Segmentation by Defining Boundary Points with Normals}

Unlike the nodes of SNAKES [Kass87], the boundary points are not required to be structured because any structural or order information is not utilized in construction of implicit function $s(\mathbf{x})$. Therefore, by using RBF approximation, interactive definition of unstructured boundary points leads to interactive segmentation with a simple inter- 
face, and automated detection of boundary points leads to fully automated segmentation. In the automated boundary point definition, gradient vectors are used as normals at the boundary.
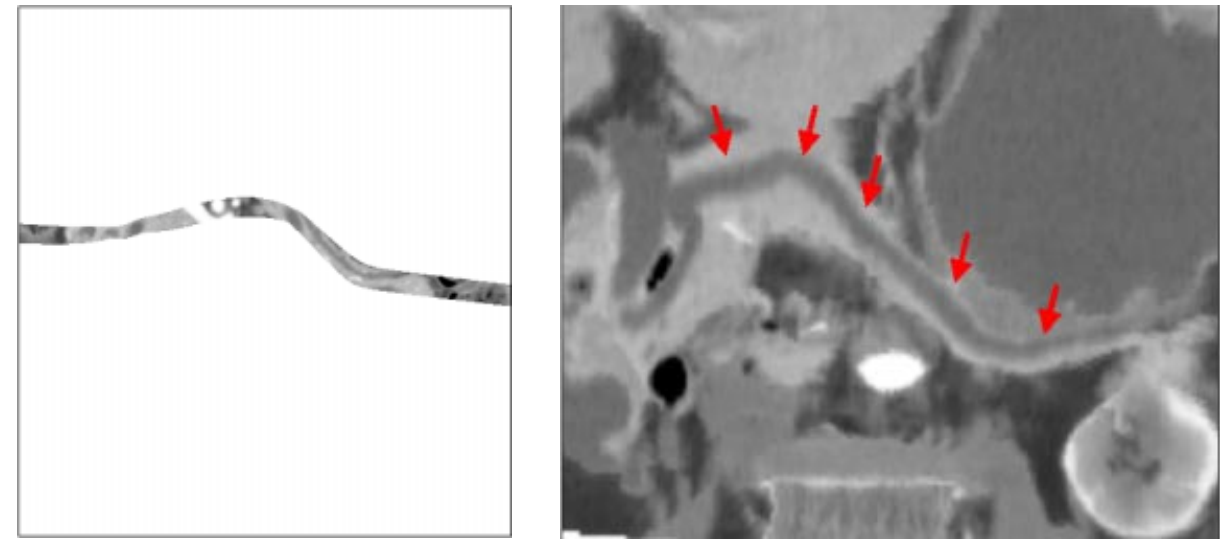

Fig. 2. Curved Thin Slab by RBF. Left: an axial slice of the reconstructed volume. Right: visualization of the main pancreatic duct by MINIP of the thin slab (arrows)

\subsubsection{Introducing a Priori Knowledge by Non-boundary Control Points}

In the RBF-based segmentation proposed in this paper, a priori knowledge is introduced by defining non-boundary control points, which are employed to suppress function values at the positions where the target object does not exist, as shown in Fig. 3. For example, in segmentation of the liver, points on other structures such as the body surface can be employed.

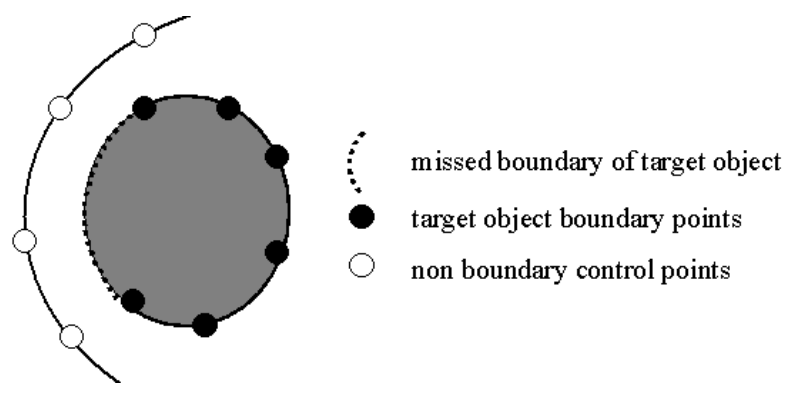

Fig. 3. Suppressing Function Value by Non-Boundary Control Points

\section{Results}

\subsection{Semi-automated Liver Segmentation}

Fig. 4 shows a result of liver segmentation based on detection of 605 boundary points. Because the algorithm of the boundary point detection in detail is out of the scope of this paper, the process is summarized briefly by the following steps: (1) presegmentation of the body surface, (2) initial segmentation of the liver by thresholding, 
(3) collecting boundary points with feature values, (4) classification of boundary points and eliminating false boundary points, if any (4) generating control points by using the selected boundary points with their normals, (5) placing non-boundary control points of the body surface, and (6) construction of implicit function by the control points.
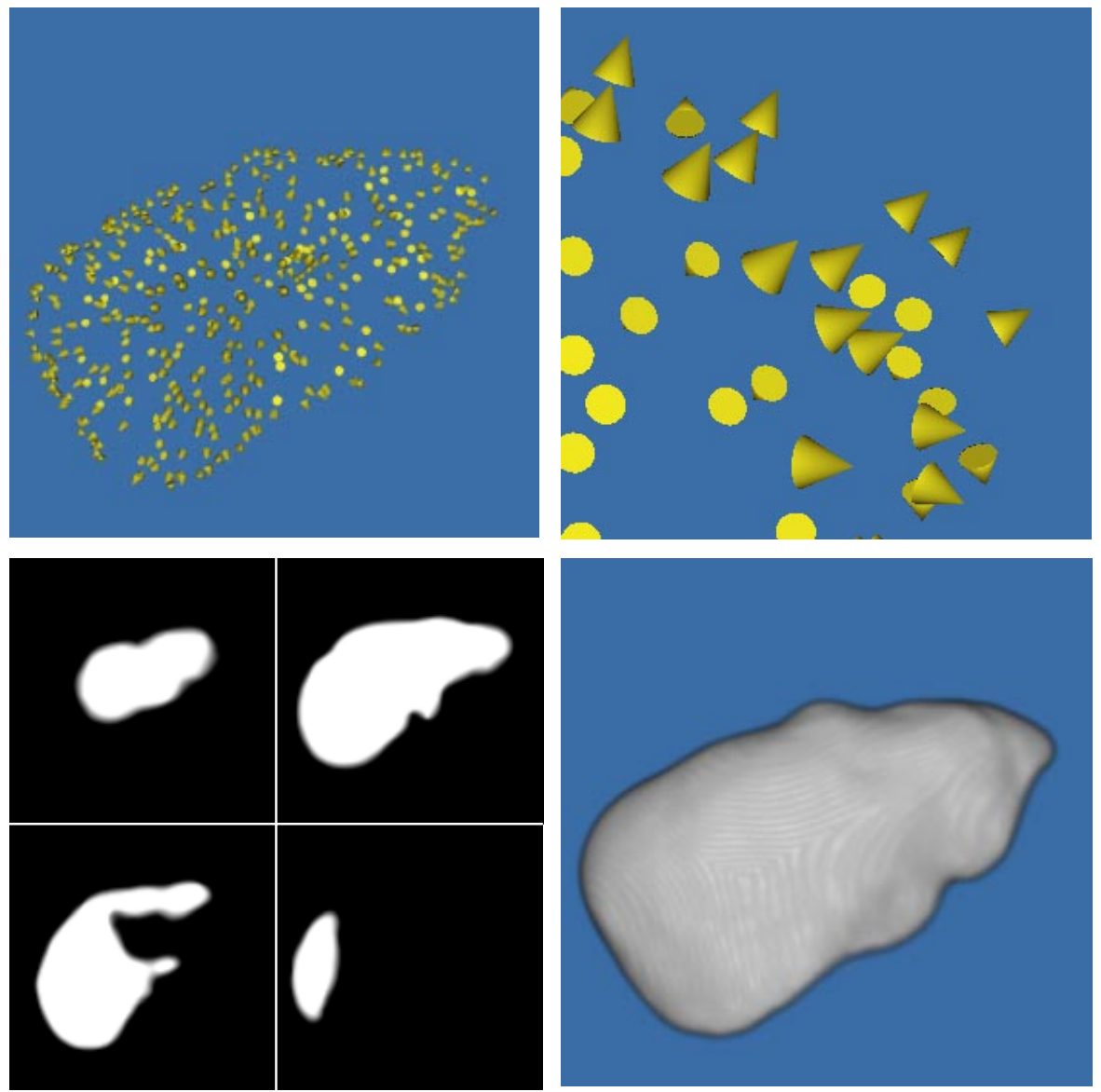

Fig 4. A Segmentation Result by Boundary Point Detection and RBF Reconstruction. Top left: detected boundary points, Top right: enlarged of Top left, the cones represent boundary points and their normal directions. Bottom left: Reconstructed volume data from the boundary points (only four slices shown). Bottom right: Reconstructed volume data visualized by volume rendering (matrix size: $128 \times 128 \times 80$ )

\subsection{Reducing Number of Boundary Points}

An important interest in the proposed segmentation method is number of the boundary points employed. Naturally, more boundary points yield more fine structures of shapes and require more computational cost. The optimal number of boundary points for object segmentation depends on complexity of object surface. Fig. 5 shows a 
result of an experiment of boundary point reduction for the liver shape segmented in the previous section. For several number of boundary points reduced, the percentages of the true positive (TP), false positive (FP), and false negative (FN) to the original liver volume were evaluated. Volume reconstruction by using only 605 points covers 97 percent of the original liver volume segmented by over 20,000 boundary points.
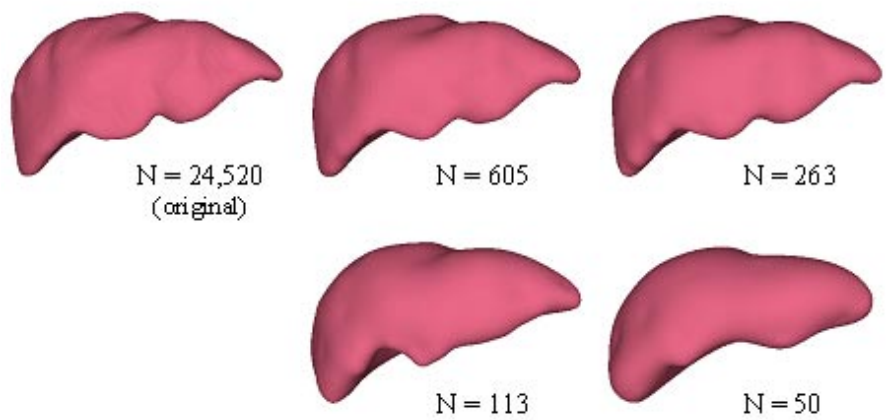

(a)

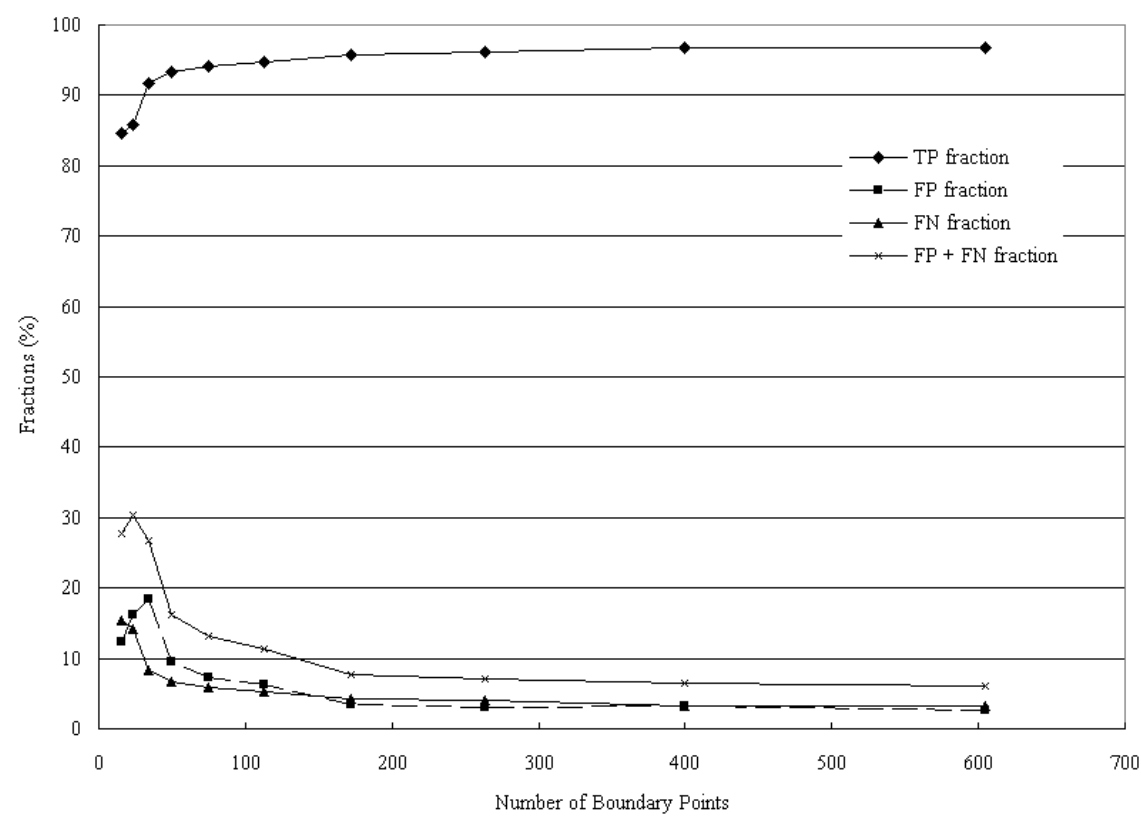

(b)

Fig. 5. Segmentation Error by Reducing Boundary Points, (a) Segmentation results with boundary points reduced, the number of boundary points: $\mathrm{N}=24,520$ is the original, results are visualized by isosurfaces. (b) Segmentation error in volumetric fractions of the true positive voxels, false positive voxels, false negative voxels 


\section{Discussion and Summary}

A new scheme of segmentation in volumetric data was proposed based on the radial basis function reconstruction. It is interesting to compare the proposed method with the method based on deformable models as shown in Table 1.

Because the proposed method is driven by data of detected boundary points, no fitting process is required. In a segmentation process, as some nodes of deformable models often miss boundary due to unsharp edge, boundary point detection is not guaranteed to be successful in the proposed method of RBF-based segmentation. In such cases of missing partial boundary, deformable models manage to interpolate the missing part based on internal energy to smooth the boundary, or is guaranteed to be smooth by defining the entire shape parametrically in frequency domain [Szekely96][Chuang96]. RBF also guarantees the smoothness of the resulting implicit function. In general, such pre-guaranteed smoothness of objects and initial shape of the models are kinds of a priori or anatomical standard information, which helps segmentation. In the proposed method, such a priori knowledge as constraint is represented more moderately to suppress implicit function values.

Apparently, the key to fully automated segmentation is methods for fully automated boundary point detection or for fully automated elimination of false boundary points. As shown in Fig. 6, false boundary points yield isolated regions when they are apart from the true boundary. In such cases, it is not hard to remove the false regions.

An important feature of the proposed method is that the control points are not structured, unlike the point distribution model (PDM) [Cootes95]. However, the notion of points with explicit features (ex. corner) might help more accurate boundary point detection. As current work, improvement of the boundary point detection based on statistical pattern analysis is in progress.

Table 1. Comparison of Proposed Method and Deformable Models

\begin{tabular}{|c|c|c|}
\hline & Proposed Method & Deformable models \\
\hline Category & Data-driven & Model-based \\
\hline $\begin{array}{l}\text { Optimization } \\
\text { process }\end{array}$ & - & Required for fitting \\
\hline $\begin{array}{c}\text { A Priori } \\
\text { Knowledge }\end{array}$ & $\begin{array}{c}\text { Features of BP } \\
\text { Non-boundary CP }\end{array}$ & $\begin{array}{c}\text { Initial shape } \\
\text { Parameter Range }\end{array}$ \\
\hline Points/Nodes & Independent & $\begin{array}{l}\text { Structured as contiguous } \\
\text { model }\end{array}$ \\
\hline Typical errors & $\begin{array}{c}\text { Isolated Artifact Region by False } \\
\text { BP, Missing BP }\end{array}$ & $\begin{array}{l}\text { Fitting to other structures, } \\
\text { Underestimation of struc- } \\
\text { tures (missing corners, etc.) }\end{array}$ \\
\hline Contiguity & No guarantee for contiguity & Compact as a model \\
\hline Result & $\begin{array}{c}\text { Implicit function } \\
\text { (Volumetric pattern) }\end{array}$ & Geometric Model \\
\hline Variation & - & $\begin{array}{c}\text { Parametric Model (Fourier, } \\
\text { Wavelet), Point Distribution } \\
\text { Model }\end{array}$ \\
\hline
\end{tabular}

BP: boundary points, $\mathrm{CP}$ : control points 

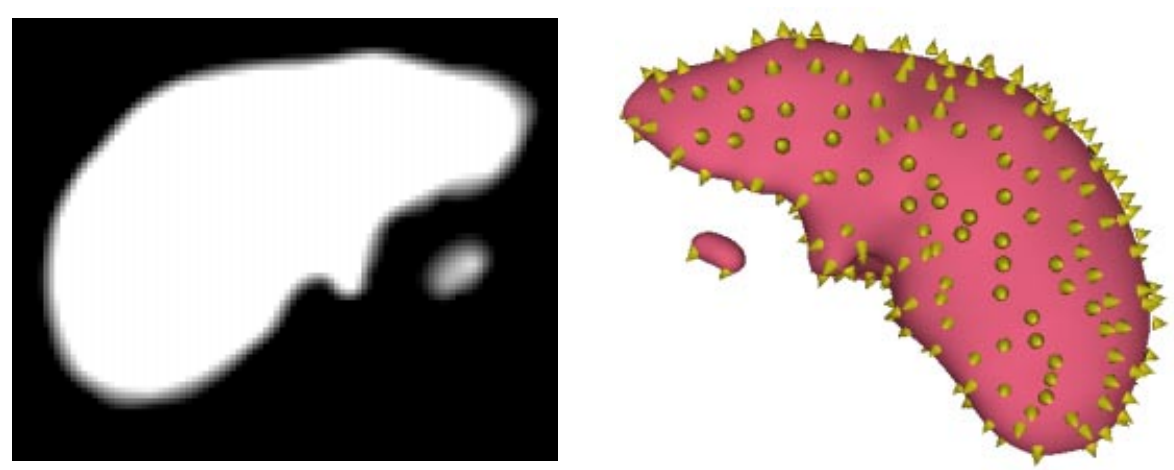

Fig. 6. An Example of Effect by False Boundary Points. Left: a slice including an isolated region, Right: an isolated region apart from the liver (normals are also shown by cones)

\section{Acknowledgements}

The author is grateful to the research groups in the laboratories of the associate professor Hiromasa Suzuki, and the professor. Fumihiko Kimura in the School of Engineering, the University of Tokyo Graduate School for the encouraging discussion in computer graphics and modeling. The author also appreciates Dr. Shigeru Kiryuu's providing the clinical pancreas CT data and advice on pancreas visualization. The clinical CT data of the liver are distributed by the Japanese society of computerassisted diagnosis in medicine (CADM) by courtesy of Dr. Shigeru Nawano.

\section{Reference}

[Kass87] Kass, M, et al., Active contour models. International Journal of Computer Vision, pages 321-331, 1987.

[Masutani01] Masutani, Y, et al., Automated Segmentation of Colonic Walls for Computerized Detection of Polyps in CT Colonography, Journal of Computer-Assisted Tomography, vol.25, no.4, pp629-638, 2001

[Savchenko95] Savchenko VV, et al.: Function Representation of Solids Reconstructed from Scattered Surface Points and Contours, Computer Graphics Forum, 14(4): 181-188, 1995

[Carr97] Carr CJ, and Fright WR, Surface Interpolation with Radial Basis Functions for Medical Imaging", IEEE Trans. on Med. Img, 16(1): pp 96-107, 1997.

[Turk99] Turk G, and O'Brien J: Shape Transformation using Variational Implicit Surfaces, ACM SIGGRAPH1999, pp.335-342, 1999

[Carr01] Carr CJ, et al.: Reconstruction and Representation of 3D Objects with Radial Basis Functions, ACM SIGGRAPH2001, pp67-76, 2001

[Szekely96] Szekely G, et al. Segmentation of 2-D and 3-D objects from MRI volume data using constrained elastic deformations of flexible Fourier contour and surface models Medical Image Analysis vol.1 no.1 pp19-34, 1996

[Chuang96] Chuang GCH, et al.Wavelet Descriptor of Planar Curves: Theory and Applications IEEE trans on Image Proc. vol.5 no.1pp.56-70 1996

[Cootes95] Cootes TF, et al.,. Active Shape Models - their training and applications. Computer Vision and Image Understanding, 61(2), January 1995. 